\title{
The use of sildenafil in severe early-onset fetal growth restriction: A case report and review of current literature
}

\author{
Geoffrey H Gordon, Danielle M Berdahl*, Kara K Hoppe and Kathleen M Antony \\ Division of Maternal-Fetal Medicine, Department of Obstetrics and Gynecology, University of Wisconsin School of Medicine and Public Health, USA
}

\begin{abstract}
Severe early-onset fetal growth restriction (FGR) complicates $0.2-0.4 \%$ of pregnancies. Current management requires a balance between the risks of prematurity with those of stillbirth. Sildenafil has been suggested as a potential drug for improving neonatal outcomes in severe early-onset FGR. Here we describe a 38-year-old gravida four with two living children born at term and one prior preterm stillbirth born at 26 weeks who presented with recurrent severe early-onset FGR. The prior preterm stillbirth was due to severe intrauterine growth restriction. With knowledge of the expected poor outcome, the patient searched investigational treatment options and requested treatment using sildenafil based upon an ongoing international randomized controlled trial. After review of institutional processes for off-label medication use in pregnancy, sildenafil was initiated but later stopped due to a placental abruption. Cesarean delivery was performed at 28 weeks due to abnormal fetal heart rate tracing. The baby's weight at birth was 550 grams and she was discharged from the neonatal intensive care unit at 94 days of life on supplemental oxygen due to bronchopulmonary dysplasia. We conclude that sildenafil remains a potential therapy for FGR, but its risks have not yet been well characterized.
\end{abstract}

\section{Introduction}

Early-onset severe FGR is diagnosed when the estimated fetal weight or abdominal circumference is less than the $3^{\text {rd }}$ percentile before 32 weeks gestation; a condition which complicates $0.2-0.4 \%$ of all pregnancies [1-3]. This has recently been distinguished from small for gestational age, defined as an estimated fetal weight below the tenth percentile [3]. Although FGR may be the result of diverse maternal or fetal conditions, early onset FGR in the absence of fetal etiologies is often attributed to poor placental development. Studies have demonstrated that the likely pathophysiology of FGR involves dysregulation of trophoblastic invasion as a result of errors in trophoblastic apoptosis and/or damage from reactive oxygen species [4].

Current management of early-onset severe FGR is limited to increased fetal surveillance. Currently, there are no evidence-based treatments for FGR once it has developed. Given that FGR fetuses have known decreased placental perfusion, treatments targeting the improvement of placental blood flow have been suggested. Although no specific treatments are currently supported based upon clinical evidence, sildenafil has shown some recent promise.

Sildenafil promotes vasodilation by inhibiting the enzyme cyclic GMP phosphodiesterase type 5, thus allowing for cyclic GMP to promote the release of nitric oxide, a potent vasodilator. In pregnancy, sildenafil has shown positive results in animal models of FGR [5-7]. $\left({ }^{* * *}\right.$ Add Spiroski) Its effects on human pregnancy are currently being investigated, and preliminary data demonstrates good maternal and fetal tolerance $[2,8]$. Here we present a case of early-onset severe FGR wherein we attempted treatment with sildenafil but ultimately discontinued treatment due to placental abruption. We will also discuss current concepts and investigations regarding the use of sildenafil as a therapeutic treatment for FGR. Written informed consent has been obtained from the patient presented here.

\section{Case}

A 38-year-old G4P2102 at 20 0/7 weeks' gestation presented to our institution for a detailed fetal anatomic examination ultrasound. Her past obstetrical history was significant for an uncomplicated fullterm delivery followed by a 26 -week stillbirth in a pregnancy affected by severe FGR and severe preeclampsia. Her evaluation following the prior stillbirth was notable for a small for gestational age placenta with a small infarction. Antiphospholipid antibody testing was negative. Her third pregnancy was an uncomplicated full-term delivery. At the time of her referral for her fourth pregnancy, her medications included prenatal vitamins, $81 \mathrm{mg}$ aspirin (for her history of early preeclampsia), and ranitidine twice daily. She was otherwise healthy.

The detailed fetal anatomic examination revealed echogenic bowel. She had negative cell-free DNA aneuploidy screening, negative infectious workup, and negative cystic fibrosis testing. She declined any invasive diagnostic testing. At her follow-up growth ultrasound at $243 / 7$ weeks' gestation, the fetus was noted to have an estimated fetal weight of $435 \mathrm{~g}$, corresponding to an estimated fetal weight below the $1^{\text {st }}$ percentile. The amniotic fluid volume was normal, but the umbilical artery (UA) systolic to diastolic (S/D) ratio was elevated, and the middle cerebral artery (MCA) pulsatility index was below the $5^{\text {th }}$ percentile. The cerebral placental ratio was 0.622 , which is less than the first percentile. Doppler interrogation of the uterine arteries revealed notching and an elevated pulsatility index.

${ }^{\star}$ Correspondence to: Danielle M Berdahl, Division of Maternal-Fetal Medicine, Department of Obstetrics and Gynecology, University of Wisconsin School of Medicine and Public Health, Madison, 1010 Mound St, McConnell Hall $4^{\text {th }}$ Floor, Madison, WI 53715, USA, Tel: 608-417-6099, Fax: 608-417-4270, E-mail: dmberdah@gundersenhealth.org

Received: May 21, 2018; Accepted: June 08, 2018; Published: June 11, 2018 
After a maternal-fetal-medicine consultation, the patient approached our perinatology group with a request for sildenafil treatment. The patient had an advanced educational degree in the health care field and had performed a literature search regarding FGR and investigational interventions. We conducted a thorough review of the literature and counseled the patient regarding the potential adverse effects of sildenafil to both herself and her fetus. Because we do not routinely offer any pharmacologic treatment of intrauterine growth restriction, we did not discuss the use of any other agents, such as $\mathrm{L}$-arginine. She persisted in her desire for sildenafil treatment and gave written consent for its off-label, non-evidence based use.

Prior to initiation, the patient underwent a follow-up ultrasound assessment at 25 3/7 weeks' gestation. Repeat ultrasonography demonstrated a persistent umbilical artery S/D ratio greater than the $95^{\text {th }}$ percentile. The patient was admitted to labor and delivery for close monitoring with the initiation of sildenafil. We planned to administer $25 \mathrm{mg}$ three times daily (the same dosing as a previous prospective case-control trial) [2] and observe the fetus and mother for at least 24 hrs for any adverse effects. A baseline maternal electrocardiogram was also obtained. Prior to the ingestion of any medication, we obtained a fetal heart rate tracing. The fetus was noted to have a spontaneous deceleration of the heart rate to 70 beats per minute for a total of 3 minutes. We therefore withheld sildenafil until a course of antenatal steroids had been administered.

The patient initiated sildenafil at $256 / 7$ weeks' gestation. Within 30 minutes of medication ingestion, the fetus was noted to have recurrent heart rate decelerations. These did resolve over the next hour. Both the mother and fetus tolerated the next 3 doses, and the patient was discharged on sildenafil at 26 0/7 weeks. On the day after discharge, the patient presented to triage with vaginal bleeding. Following a thorough assessment of the patient and fetus, a diagnosis of partial placental abruption was made and the sildenafil was stopped. The patient had no further episodes of bleeding, but she remained admitted to the hospital until delivery. She was monitored with non-stress testing three times daily, as well as UA and MCA Doppler studies twice weekly. At 27 0/7 weeks, ultrasonographic assessment of the fetus revealed an estimated fetal weight of $515 \mathrm{~g}\left(<1^{\text {st }}\right.$ percentile $)$ with a persistently elevated UA S/D ratio. An area suspicious for fibrin deposition within the placenta was also noted on ultrasound (Figure 1).

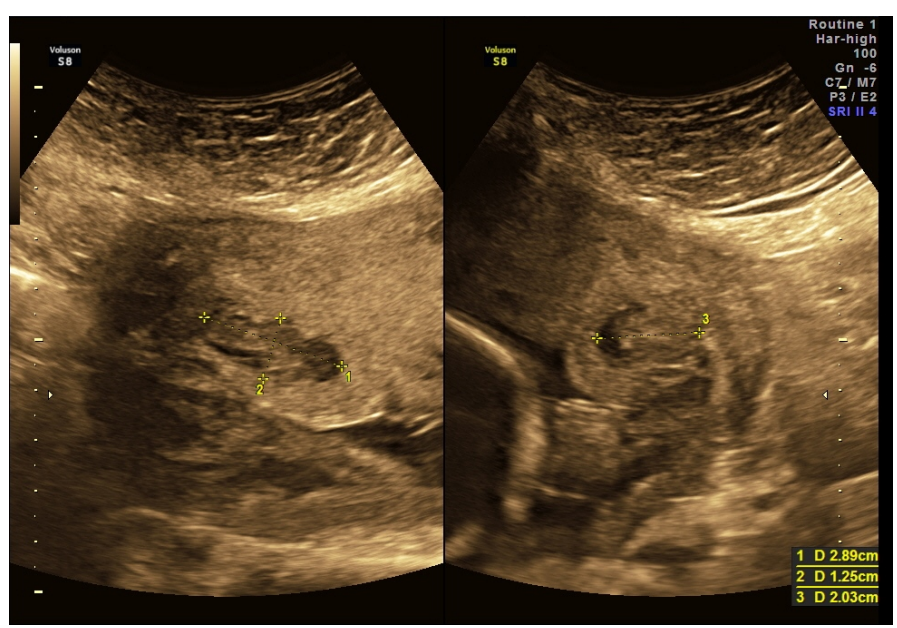

Figure 1. Transabdominal ultrasonography at $270 / 7$ weeks' gestation. The anterior placenta demonstrates an area of fibrin deposition. This was positively correlated with placental pathology after delivery
At 27 3/7 weeks, the fetal heart rate tracing demonstrated recurrent variable decelerations, and a rescue course of antenatal steroids was administered, along with magnesium sulfate for fetal neuroprophylaxis. After the tracing improved, the magnesium sulfate was discontinued. At $280 / 7$ weeks, the fetal heart tracing demonstrated recurrent decelerations (4 within a 15-minute period) from a baseline of 150 beats per minute to 40 beats per minute, with a return to baseline of 1-3 minutes each. Magnesium sulfate was restarted, and a live born female neonate weighing $550 \mathrm{~g}$ with APGARS of 7 and 9 was delivered by Cesarean. Due to the small size of the cord, an adequate sample for cord gases was unable to be obtained. The placenta weighed 140 $\mathrm{g}\left(\sim 10^{\text {th }}\right.$ percentile $)$ with distal villous hypoplasia with areas of patchy fibrotic villi. Fresh and remote infarcts were also present, as was maternal decidual vasculopathy. All findings were consistent with a chronic uterine malperfusive state. The baby was discharged from the neonatal intensive care unit at 94 days on supplemental oxygen due to bronchopulmonary dysplasia.

\section{Discussion}

The implications of FGR extend well beyond the immediate concern of fetal compromise and stillbirth. Aside from the acute prematurity related concerns of respiratory distress, necrotizing enterocolitis, and retinopathy of prematurity, the long-term consequences of FGR include increased risks for diabetes, obesity, and cardiovascular disease [9].

Sildenafil acts as a vasodilator by inhibiting the enzyme cyclic GMP phosphodiesterase type 5 and thus prolongs nitric oxide's effect. In animal studies and animal pregnancy and embryo models, sildenafil seems to exhibit immunomodulatory effects, increase angiogenesis, increase nitric oxide bioavailability, and reduce oxidative stress [1012]. Animal models have also demonstrated that sildenafil treatment improves placental weight, decreases placental resistance, and may alter cerebral redistribution $[6,13]$.

Sildenafil has been shown to produce a reduction in vasoconstriction and promote improved vessel relaxation when exposed to small arteries from myometrial biopsies of pregnancies complicated by FGR $[7,14]$. More recently, the use of sildenafil on first-trimester villous explants suggests that it has the potential to increase the trophoblastic invasiveness [15]. Recent studies have also demonstrated that sildenafil citrate can improve uteroplacental blood flow in pregnancies with FGR, and future studies may show efficacy in treating fetal growth restriction $[7,16]$.

Our patient did not have a clear etiology for FGR; unlike in her prior pregnancy, she never developed preeclampsia. However, there was a large fibrin deposit noted in the placenta on both ultrasound and gross pathology, which has clinically been associated with severe FGR and fetal demise and histopathologically associated with both maternal floor infarctions as well as massive chorionic intervillositis [17-21].

Once we identified sildenafil as a potential therapeutic option for severe early-onset FGR, we analyzed the available literature and identified an ongoing series of prospective trials called "The Sildenafil Therapy In Dismal Prognosis Early-Onset Intrauterine Growth Restriction (STRIDER) Trial" [1]. Although our patient met the listed inclusion criteria for the study, she was not able to travel outside the United States and therefore, this precluded her from enrolling. We were faced with a dilemma of a patient requesting a treatment that is outside the standard of care, yet currently under investigation with some potential efficacy. We held a conference amongst our division of maternal-fetal-medicine physicians to discuss her case. With the 
assistance of our hospital legal team, a detailed consent form was established, and the patient was counseled extensively on the risks and unclear benefits, as well as the plethora of unknowns prior to signing this form. The discussion and consent form detailed all known and potential side effects of sildenafil in general, such as cardiovascular symptoms, central nervous system symptoms, and gastrointestinal effects. The consent form also discussed the specific concerns of using this medication during pregnancy. We discussed the potential to worsen the fetal condition or result in unknown long-term fetal effects or death. We also discussed that this medication would preferably be used in a research setting and we discussed that there were research sites in Europe and Australia. There are now also research sites in Canada, but that was not the case when this patient presented. We also discussed that her insurance would likely not cover the charges of this medication, given its use for an off-label indication for which there was very limited evidence in the literature.

Small published trials and case series utilizing sildenafil for preeclampsia or oligohydramnios indicate that both mothers and fetuses tolerate the medication, and that it may prolong pregnancy in select cases $[4,8,22-26]$. Specifically in regards to severe early-onset fetal growth restriction, there have been case reports and one prospective casecontrol trial using sildenafil as a treatment $[2,26,27]$. The small cohort of women who received sildenafil $(n=10)$ did show an improvement in fetal abdominal circumference measurements compared to the sildenafil naive cohort. However, this study was underpowered to detect any significant changes in long-term outcomes regarding morbidity or mortality of neonates. Subsequent meta-analyses have also supported the use of sildenafil on improving fetal growth in cases of FGR, although the overall number of patients remains low $[28,29]$.

Understanding that sildenafil may cause changes in our patient's physiology (potential hypotension and reflexive tachycardia), we opted for inpatient medication initiation to monitor the tolerance of the mother. Furthermore, although sildenafil has been documented to be tolerated well by the fetus, we opted to monitor the fetus for at least one hour after administration to ensure fetal tolerance.

It is unclear whether or not sildenafil contributed to our patient's placental abruption. However, given the mechanism of sildenafil and the likelihood that this medication caused increased blood flow to the uteroplacental interface, it is possible that this may have caused more damage to an already fragile villous network. The placenta and fetus in severe FGR exist in chronic states of hypoxia. Vasodilation may make the placenta susceptible to rapid increase in oxygenation and the production of reactive oxygen species. This may worsen the anchoring ability of the villi and increase the risk of abruption.

It is yet to be determined if sildenafil will have a positive impact on the outcomes of severe early-onset FGR. Since the time that this patient presented, one of the STRIDER randomized controlled trials published its results. It found that sildenafil failed to prolong pregnancy or improve outcomes in early FGR [30]. This study further recommended that sildenafil should not be prescribed for FGR outside of a research study with explicit patient consent. Notably, this trial did not report on placental abruption as an outcome; future published trials may report this.

We acknowledge that our case stands out against other publications due to the discontinuation of sildenafil due to a possible placental abruption. However, as we continue to understand FGR and its etiology, it may become clear that new innovative therapies do not ameliorate FGR in all patient subsets. The use of sildenafil in this case may have been initiated too late to reverse the pathologic placental changes. Regardless, we patiently await the results of the ongoing randomized trials, which will hopefully identify a potential therapy for severe early onset intrauterine growth restriction.

\section{References}

1. Ganzevoort W, Alfirevic Z, von Dadelszen P, Kenny L, Papageorghiou A, et al. (2014) STRIDER: Sildenafil Therapy In Dismal prognosis Early-onset intrauterine growth Restriction--a protocol for a systematic review with individual participant data and aggregate data meta-analysis and trial sequential analysis. Syst Rev 3: 23. [Crossref]

2. Von Dadelszen P, Dwinnell S, Magee LA, et al. (2011) Sildenafil citrate therapy for severe early-onset intrauterine growth restriction. BJOG An Int J Obstet Gynaecol 118: 624-628.

3. Gordijn SJ, Beune IM, Thilaganathan B, et al. (2016) Consensus definition of fetal growth restriction: a Delphi procedure. Ultrasound Obstet Gynecol 48: 333-339. [Crossref]

4. Scifres CM, Nelson DM (2009) Intrauterine growth restriction, human placental development and trophoblast cell death. J Physiol 587: 3453-3458. [Crossref]

5. Dilworth MR, Andersson I, Renshall LJ, Cowley E, Baker P, et al. (2013) Sildenafil Citrate Increases Fetal Weight in a Mouse Model of Fetal Growth Restriction with a Normal Vascular Phenotype. PLoS One 8: e77748. [Crossref]

6. Oyston C, Stanley JL, Oliver MH, Bloomfield FH, Baker PN (2016) Materna administration of sildenafil citrate alters fetal and placental growth and fetal-placental vascular resistance in the growth-restricted ovine fetus. Hypertension 68: 760-767. [Crossref]

7. Spiroski AM, Oliver MH, Harding JE, Bloomfield FH (2016) Intrauterine Intervention for the Treatment of Fetal Growth Restriction. Curr Pediatr Rev 12: 168-178. [Crossref]

8. Samangaya RA, Mires G, Shennan A, et al. (2009) A randomised, double-blinded, placebo-controlled study of the phosphodiesterase type 5 inhibitor sildenafil for the treatment of preeclampsia. Hypertens pregnancy 28: 369-382. [Crossref]

9. Longo S, Bollani L, Decembrino L, Di Comite A, Angelini M, et al. (2013) Shortterm and long-term sequelae in intrauterine growth retardation (IUGR). J Matern Fetal Neonatal Med 26: 222-225. [Crossref]

10. Ölmestig JNE, Marlet IR, Hainsworth AH, Kruuse C (2017) Phosphodiesterase 5 inhibition as a therapeutic target for ischemic stroke: A systematic review of preclinical studies. Cell Signal 38: 39-48. [Crossref]

11. Kniotek M, Boguska A (2017) Sildenafil Can Affect Innate and Adaptive Immune System in Both Experimental Animals and Patients. J Immunol Res 2017: 4541958. [Crossref]

12. Itani N, Skeffington KL, Beck C, Giussani DA (2017) Sildenafil therapy for fetal cardiovascular dysfunction during hypoxic development: studies in the chick embryo. $J$ Physiol 595: 1563-1573. [Crossref]

13. López-Tello J, Arias-Álvarez M, Jiménez-Martínez MÁ, et al. (2017) The effects of sildenafil citrate on feto-placental development and haemodynamics in a rabbit model of intrauterine growth restriction. Reprod Fertil Dev 29: 1239-1248. [Crossref]

14. Wareing M, Myers JE, O’Hara M, Baker PN (2005) Sildenafil citrate (viagra) enhances vasodilatation in fetal growth restriction. J Clin Endocrinol Metab 90: 2550-2555.

15. Bolnick JM, Kilburn BA, Bolnick AD, et al. (2015) Sildenafil stimulates human trophoblast invasion through nitric oxide and guanosine 3',5'-cyclic monophosphate signaling. Fertil Steril 103: 1582-1587. [Crossref]

16. El-Sayed MA, Saleh SAA, Maher MA, Khidre AM (2018) Utero-placental perfusion Doppler indices in growth restricted fetuses: effect of sildenafil citrate. J Matern Neonatal Med 31: 1045-1050. [Crossref]

17. Feist H, Blöcker T, Hussein K (2015) Massive perivillöse Fibrinabscheidungen, chronisch-histiozytäre Intervillositis, Villitis unbekannter Ätiologie: Plazentaläsionen bei Störungen der fetomaternalen Einheit mit Rezidivrisiko. Pathologe 36: 355-361.

18. Weber MA, Nikkels PGJ, Hamoen K, Duvekot JJ, de Krijger R (2006) Co-Occurrence of Massive Perivillous Fibrin Deposition and Chronic Intervillositis: Case Report. Pediatr Dev Pathol 9: 234-238. [Crossref]

19. Marchaudon V, Devisme L, Petit S, Ansart-Franquet H, Vaast P, et al. (2011) Chronic histiocytic intervillositis of unknown etiology: Clinical features in a consecutive series of 69 cases. Placenta 32: 140-145. [Crossref]

20. Man J, Hutchinson JC, Heazell AE, Ashworth M, Jeffrey I, et al. (2016) Stillbirth and intrauterine fetal death: role of routine histopathological placental findings to determine cause of death. Ultrasound Obstet Gynecol 48: 579-584. [Crossref] 
21. Levytska K, Higgins M, Keating S, Melamed N, Walker M, et al. (2017) Placental Pathology in Relation to Uterine Artery Doppler Findings in Pregnancies with Severe Intrauterine Growth Restriction and Abnormal Umbilical Artery Doppler Changes. $\mathrm{Am}$ J Perinatol 34: 451-457. [Crossref]

22. Trapani A, Gonçalves LF, Trapani TF, Vieira S, Pires M, et al. (2016) Perinatal and Hemodynamic Evaluation of Sildenafil Citrate for Preeclampsia Treatment. Obste Gynecol 128: 253-259. [Crossref]

23. Trapani A, Gonçalves LF, Trapani TF, Franco MJ, Galluzzo RN, et al. (2016) Comparison between transdermal nitroglycerin and sildenafil citrate in intrauterine growth restriction: effects on uterine, umbilical and fetal middle cerebral artery pulsatility indices. Ultrasound Obstet Gynecol 48: 61-65. [Crossref]

24. Maher MA, Sayyed TM, Elkhouly N (2017) Sildenafil Citrate Therapy for Oligohydramnios: A Randomized Controlled Trial. Obstet Gynecol 129: 615-620. [Crossref]

25. Dunn L, Greer R, Flenady V, Kumar S (2017) Sildenafil in Pregnancy: A Systematic Review of Maternal Tolerance and Obstetric and Perinatal Outcomes. Fetal Diagn Ther 41: 81-88. [Crossref]
26. Sakamoto M, Osato K, Kubo M, Nii M, Tanaka H, et al. (2016) Early-onset fetal growth restriction treated with the long-acting phosphodiesterase-5 inhibitor tadalafil: a case report. J Med Case Rep 10: 1-5. [Crossref]

27. Choudhary R, Desai K, Parekh H, Ganla K (2016) Sildenafil citrate for the management of fetal growth restriction and oligohydramnios. Int $J$ Womens Health 8: 367-372. [Crossref]

28. Chen J, Gong X, Chen P, Luo K, Zhang X (2016) Effect of L-arginine and sildenafil citrate on intrauterine growth restriction fetuses: A meta-analysis. BMC Pregnancy Childbirth 16: 1-7. [Crossref]

29. Paauw ND, Terstappen F, Ganzevoort W, Joles JA, Gremmels H, et al. (2017) Sildenafil During Pregnancy: A Preclinical Meta-Analysis on Fetal Growth and Maternal Blood Pressure. Hypertension 70: 998-1006. [Crossref]

30. Sharp A, Cornforth C, Jackson R, et al. (2018) Maternal sildenafil for severe fetal growth restriction (STRIDER): a multicentre, randomised, placebo-controlled, doubleblind trial. Lancet Child Adolesc Heal 2: 90.

Copyright: $\odot 2018$ Gordon GH. This is an open-access article distributed under the terms of the Creative Commons Attribution License, which permits unrestricted use, distribution, and reproduction in any medium, provided the original author and source are credited. 